Stasinakis, C., Sermpinis, G., Theofilatos, K., and Karathanasopoulos, A. (2015) Forecasting US unemployment with radial basis neural networks, kalman filters and support vector regressions. Computational Economics.

Copyright (C 2014 Springer Science+Business Media New York

A copy can be downloaded for personal non-commercial research or study, without prior permission or charge

Content must not be changed in any way or reproduced in any format or medium without the formal permission of the copyright holder(s)

When referring to this work, full bibliographic details must be given

http://eprints.gla.ac.uk/102080

Deposited on: 03 February 2015

Enlighten - Research publications by members of the University of Glasgow_ http://eprints.gla.ac.uk 


\title{
Forecasting US unemployment with Radial Basis Neural Networks, Kalman Filters and Support Vector Regressions
}

\author{
Charalampos Stasinakis* \\ Georgios Sermpinis** \\ Konstantinos Theofilatos*** \\ Andreas Karathanasopoulos****
}

* Corresponding Author, University of Glasgow Business School, University of Glasgow, Adam Smith Building, Glasgow, G12 8QQ, UK (Charalampos.Stasinakis@glasgow.ac.uk)

**University of Glasgow, Business School, University of Glasgow, Adam Smith Building, Glasgow, G12 8QQ, UK (georgios.sermpinis@glasgow.ac.uk)

***Pattern Recognition Laboratory, Dept. of Computer Engineering \& Informatics, University of Patras, 26500, Patras, Greece (theofilk@ceid.upatras.gr) **** University of East London, Royal Docks Business School, University Way, London, E16 2RD, UK (a.karathanasopoulos@uel.ac.uk) 


\begin{abstract}
This study investigates the efficiency of the Radial Basis Function Neural Networks in forecasting the US unemployment and explores the utility of Kalman Filter and Support Vector Regression as forecast combination techniques. On one hand, an Autoregressive Moving Average model, a Smooth Transition Autoregressive Model and three different Neural Networks architectures, namely a Multi-Layer Perceptron, Recurrent Neural Network and a Psi Sigma Network are used as benchmarks for our Radial Basis Function Neural Network. On the other hand, our forecast combination methods are benchmarked with a Simple Average and a Least Absolute Shrinkage and Selection Operator. The statistical performance of our models is estimated throughout the period of 1972-2012, using the last seven years for out-of-sample testing. The results show that the Radial Basis Function Neural Network statistically outperforms all models' individual performances. The forecast combinations are successful, since both Kalman Filter and Support Vector Regression techniques improve the statistical accuracy. Finally, Support Vector Regression is found to be the superior model of the forecasting competition. The empirical evidence of this application is further validated by the use of the modified Diebold Mariano test.
\end{abstract}


Keywords: Forecast Combinations, Kalman Filter, Support Vector Regression, Unemployment

\section{Introduction}

The voluminous macroeconomic literature includes a variety of forecasting competitions of linear and non-linear architectures. Through these studies researchers attempt to shed light on time series, such as inflation or unemployment, that are relevant to monetary and policy decisions worldwide. Several techniques have been applied to such forecasting tasks with ambiguous results. Therefore, statisticians and econometricians turn to highly computational, time-varying and adaptive in nature techniques. Neural networks (NNs) are one such class of models that can assist their quest for improved forecast accuracy. Especially in periods of extreme structural instabilities, NNs' data-adaptive learning and clustering ability can prove to be very useful in forecasting applications (Zhang et al. 1998). It is, thus, not surprising that NNs continue to receive a great deal of attention in the literature (Huang et al. 2013; Özkan 2013; Fernandes et al. 2014; Olmedo 2014).

Forecasting unemployment rates, especially, is a very well documented case study (Szpiro 1997; Montgomery et al. 1998; Rothman 1998; Koop and Potter 1999). Skalin and Teräsvirta (2002) use multivariate STAR models to forecast unemployment rates. Moshiri and Brown (2004) apply a back-propagation model and a generalized regression NN model to estimate post-war aggregate unemployment rates in the USA, Canada, UK, France and Japan. The out-of-sample results confirm the forecasting superiority of the NN approaches against traditional linear and non-linear autoregressive models. Bayesian NNs are applied in the case study of forecasting unemployment in West Germany by Liang (2005). The empirical evidence indicate that the NNs present significantly better forecasts than traditional autoregressive models. Milas and Rothman (2008) use smooth transition vector error-correction models to predict unemployment rates in the non-Euro G7 countries. The proposed model outperforms the linear autoregressive benchmark and improves significantly the forecasts of the US and UK unemployment rate during business cycle expansions. Olmedo (2014) performs a competition between non-linear models, including NNs and Nearest Neighbour algorithms, to forecast different European unemployment rate time series. The best results are provided by a vector autoregressive 
and baricentric predictor. As the forecasting horizon lengthens the performance deteriorates and in some cases NNs.

The idea of combining forecasts to improve forecast accuracy is not new (Bates and Granger 1969; Newbold and Granger 1974; Deutsch et al. 1994). Swanson and Zeng (2001) perform forecast combinations based on a model-selection approach and suggest that a SIC-based approach to combine forecasts can be a useful alternative to combination methods such as simple averaging or mean square error minimization. Teräsvirta et al. 2005 examine the forecast accuracy of linear autoregressive, smooth transition autoregressive and $\mathrm{NN}$ models for 47 monthly macroeconomic variables, including unemployment rates, of the G7 economies. The empirical results prove that their forecasting ability is much improved when they are combined with autoregressive models. Kapetanios et al. (2008) report that combinations of statistical forecasts from several models (random walks, STARs, ARs, VARs etc.) generate good forecasts of inflation and growth. They also note that such forecast combinations can serve as an unbiased benchmark, which could be compared with conditional and judgemental policymaker's expectations. Finally, Vasnev et al. (2013) combine forecasts of models incorporating monthly and quarterly macroeconomic time series to predict the monetary operations of the Reserve Bank of Australia. Their findings confirm the benefits of forecast combination models and present alternative methods of forecasting monetary decisions.

Given the previous framework, the rational of this paper is twofold. Firstly, we investigate the efficiency of the Radial Basis Function Neural Networks (RBFNNs) in forecasting the US unemployment. Secondly, we explore the utility of Kalman Filter and Support Vector Regression (SVR) as forecast combination techniques. On one hand, an Autoregressive Moving Average model (ARMA), a Smooth Transition Autoregressive Model (STAR) and three different Neural Networks architectures, namely a Multi-Layer Perceptron (MLP), Recurrent Neural Network (RNN) and a Psi Sigma Network (PSN) are used as benchmarks for our RBFNN. On the other hand, our forecast combination methods are benchmarked with a Simple Average and a Least Absolute Shrinkage and Selection Operator (LASSO). The statistical performance of our models is estimated throughout the period of 1972-2012, using the last seven years for out-of-sample testing. 
The empirical evidence of this application is further validated by the use of the modified Diebold Mariano test.

With this study, we intend to extend the growing literature of using RBFNNs and NNs in general in financial and macroeconomic forecasting task. In addition, the evaluation of the Kalman Filter and SVR adds validity to the evidence of previous studies that report the benefits of combining forecasts. Finally, the performance of those non-linear and time-varying combination methods evaluate if there is a need to experiment beyond traditional linear equivalents.

The rest of the paper is organized as follows. Section 2 presents the description of the dataset used in this application. Sections 3 and 4 give an overview of the forecasting models and the forecast combination methods implemented respectively. The statistical performance of our models is presented in Section 5. Finally, some concluding remarks are summarized in Section 6.

\section{US Unemployment Dataset}

In this study, we forecast the monthly change of the US unemployment rate (UNEMP). The data can be found on the online Federal Reserve Economic Data (FRED) database of the Federal Reserve Bank of St. Louis ${ }^{1}$. This forecasting exercise explores the performance of the models over the period of 1972 to 2012, using the last seven years for out-of-sample evaluation. The time series is seasonally adjusted. For training purposes of our NNs, we further divide our in-sample dataset in two sub-periods; the training and test sub-period (see section 3.3). The total dataset is summarized in Table 1 below.

\section{[Insert Table 1]}

The following graph presents the US unemployment rate for the period under study.

\section{[Insert Figure 1]}

\footnotetext{
${ }^{1}$ The US unemployment rate or civilian unemployment rate represents the number of unemployed as a percentage of the labour force. Labour force data are restricted to people 16 years of age and older, who currently reside in 1 of the 50 states or the District of Columbia, who do not reside in institutions (e.g., penal and mental facilities, homes for the aged) and who are not on active duty in the Armed Forces. This is the definition provided by FRED.
} 
In the literature, there is no formal theory behind the selection of the inputs of a NN. Therefore, we conduct some NN experiments and a sensitivity analysis on a pool of potential inputs in the in-sample dataset in order to help our decision. The set of inputs that provide the best statistical performance for each network in the test sub-period are finally retained. In this case, those sets of inputs are autoregressive terms of UNEMP ${ }^{2}$ and are presented in Table 2 below:

[Insert Table 2]

\section{Forecasting Models}

\subsection{Auto-Regressive Moving Average Model (ARMA)}

The ARMA model is used to benchmark the efficiency of the NNs' statistical performance. Using as a guide the correlogram and the information criteria in the insample subset, we have chosen a restricted ARMA $(7,7)$ model, where all the coefficients are significant at the 95\% confidence interval. The selected ARMA model is presented in equation (1) below:

$$
\hat{Y}_{t}=0.03+1.025 Y_{t-1}-0.293 Y_{t-2}+0.511 Y_{t-4}-0.321 Y_{t-7}-1.006 \varepsilon_{t-1}+0.463 \varepsilon_{t-2}-0.545 \varepsilon_{t-4}-0.211 \varepsilon_{t-7}
$$

where $\hat{Y}_{t}$ is the forecasted monthly change of the US unemployment rate.

\subsection{Smooth Transition Autoregressive Model (STAR)}

STARs initially proposed by Chan and Tong (1986) are extensions of the traditional autoregressive models (ARs). The STAR combines two AR models with a function that

\footnotetext{
${ }^{2}$ We also explored autoregressive terms of other US macroeconomic indicators (e.g. the Consumer Price Index, the Industrial Production Index, M1 money stock) as potential inputs. However, the set of inputs presented in Table 2 gave our NNs the best statistical performance in the test sub- period during our sensitivity analysis.
} 
defines the degree of non-linearity (smooth transition function). The general two-regime STAR specification is the following:

$$
\hat{Y}_{t}=\Phi_{1}^{\prime} \mathrm{X}_{t}\left(1-F\left(z_{t}, \zeta, \lambda\right)\right)+\Phi_{2}^{\prime} \mathrm{X}_{t} F\left(z_{t}, \zeta, \lambda\right)+u_{t}
$$

Where:

- $\quad \hat{Y}_{t}$ the forecasted value at time $t$

- $\Phi_{i}=\left(\tilde{\varphi}_{i, 0}, \tilde{\varphi}_{i, 1}, \ldots \tilde{\varphi}_{i, p}\right), i=1,2$ and $\tilde{\varphi}_{i, 0}, \tilde{\varphi}_{i, 1}, \ldots \tilde{\varphi}_{i, p}$ the regression coefficients of the two AR models

- $\quad \mathrm{X}_{t}=\left(1, \tilde{\chi}_{t}^{\prime}\right)^{\prime}$ with $\tilde{\chi}_{t}^{\prime}=\left(Y_{t-1}, \ldots, Y_{t-p}\right)$

- $\quad 0 \leq F\left(z_{t}, \zeta, \lambda\right) \leq 1$ the smooth transition function

- $\quad z_{t}=Y_{t-d}, d>0$ the lagged endogenous transition variable

- $\zeta$ the parameter that defines the smoothness of the transition between the two regimes

- $\quad \lambda$ the threshold parameter

- $\quad u_{t}$ the error term

In this paper we follow the steps of Lin and Teräsvirta (1994) in order to determine when the series is best modeled as a Logistic STAR or an Exponential STAR process. In our case, the series is modeled as an Exponential one.

\subsection{Neural Networks (NNs)}

\subsubsection{NN Benchmarks}

The use of NNs in financial and macroeconomic forecasting is not new, since researchers use them to identify patterns and exploit their adaptive nature in relevant time series (Hiemstra 1996; Moshiri et al. 1999; Zhang and Qi 2005). In this study, three NNs 
architectures, namely the MLP, RNN and the PSN are applied to the task of forecasting US unemployment rate and act as NN benchmarks to the RBFNN.

These three architectures have at least three layers. The first layer is called the input layer (the number of its nodes corresponds to the number of explanatory variables). The last layer is called the output layer (the number of its nodes corresponds to the number of response variables). An intermediary layer of nodes, the hidden layer, separates the input from the output layer. Its number of nodes defines the amount of complexity the model is capable of fitting. In addition, the input and hidden layer contain an extra node called the bias node. This node has a fixed value of one and has the same function as the intercept in traditional regression models. Normally, each node of one layer has connections to all the other nodes of the next layer. The training of the network (which is the adjustment of its weights in the way that the network maps the input value of the training data to the corresponding output value) starts with randomly chosen weights and proceeds by applying a learning algorithm called backpropagation of errors (Shapiro 2000). The iteration length is optimised by maximising a fitness function in the test dataset.

Unlike MLPs, RNNs have an activation feedback which embodies short-term memory. In other words, the RNN architecture can provide more accurate outputs because the inputs are (potentially) taken from all previous values. Tenti (1996) reports that they need more connections and memory than standard back-propagation networks, but they can yield better results in comparison with simple MLPs due to the additional memory inputs. The PSN model was firstly introduced by Shin and Ghosh (1991). They are a class of feedforward fully connected higher order NNs, which require less number of weights and processing units for their training. Their main advantage is that they combine the fast learning property of single layer networks with the powerful mapping capability of higher order NNs, while avoiding the combinatorial increase in the required number of weights. The order of the network in the context of PSNs is represented by the number of hidden nodes. In a PSN the weights from the hidden to the output layer are fixed to one and only the weights from the input to the hidden layer are adjusted, something that greatly reduces the training time. The activation function of the nodes in the hidden layer is the summing function, while the activation function of the output layer is a sigmoid one. For more information on MLP, RNN and PSN architectures see Zhang et al. (1998) and 
Sermpinis et al. (2012). The summary of the structure and the training characteristics of those networks are presented in the Appendix A.

\subsubsection{Radial Basis Function Neural Networks (RBFNN)}

Initially proposed by Broomhead and Lowe (1988), the RBFNNs are feed-forward NNs. Unlike MLP, RNN and PSN, the hidden layer of the RBFNN uses a radial basis function. RBFNNs require less training time, but they can achieve higher levels of accuracy than traditional feed-forward NNs. This is achieved through the superposition of nonorthogonal, radially symmetric functions. The following figure shows the general structure of a RBFNN.

\section{[Insert Figure 2]}

Where:

- $\quad x_{t}(n=1,2, \cdots, N+1)$ are the inputs

- $\tilde{y}_{t}$ is the output

- $w_{t}^{[j]}(j=1,2)$ are the adjustable weights

- $(3)$ is the Gaussian function: $\phi^{[i]}\left(x_{t}\right)=e^{-\frac{\left\|x_{t}-C_{i}\right\|^{2}}{2 \sigma_{i}^{2}}}$

- $\bigcirc$ is the linear output function: $F(\phi)=\sum_{i} \phi^{[i]}$

In order to define the Gaussian function, we need the two parameters $C_{i}$ and $\sigma_{i}$. The first one corresponds to the vector indicating the center of the function, while the second one its width. These two parameters along with the adjustable weights are optimized through the learning phase of the training of the RBFNN. Given the target value $y_{t}$ and the number of iterations $\mathrm{T}$, the error function to be minimized is:

$$
E\left(C, \sigma, w_{t}\right)=\frac{1}{T} \sum_{t=1}^{T}\left(y_{t}-\tilde{y}_{t}\left(w_{t}, C, \sigma\right)\right)^{2}
$$


The training characteristics of RBFNN are also presented in Appendix A.

\section{Forecast Combination Techniques}

All the forecast combination techniques implemented in this paper are presented in this section. The traditional models of ARMA and STAR present a considerably worse statistical performance than their NNs' counterparts both in-sample and out-of-sample. Therefore, we decided to exclude them from our forecast combination procedures.

\subsection{Simple Average}

As a benchmark for the other three, more sophisticated, forecast combination methods, we use a simple average of the four individual forecasts of MLP, RNN, PSN and RBFNN. Thus, given the forecasts $f_{M L P}^{t}, f_{R N N}^{t}, f_{P S N}^{t}, f_{R B F N N}^{t}$ the combination forecast at time $t$ is calculated as follows:

$$
f_{c_{N N S}}^{t}=\left(f_{M L P}^{t}+f_{R N N}^{t}+f_{P S N}^{t}+f_{R B F N N}^{t}\right) / 4
$$

\subsection{Least Absolute Shrinkage and Selection Operator (LASSO)}

The LASSO method is a class of Shrinkage Regressions, which minimizes the residual squared error by adding a coefficient constraint (Sundberg, 2006). This is a similar approach to Ridge Regression (Chan et al., 1999). According to Hastie et al. 2009, though, LASSO should be selected when the used sample consists off few variables with medium/large effect, as in our exercise. 
Given the following vectors of independent and dependent variables:

$$
\left(\begin{array}{c}
X_{1}^{T} \\
\vdots \\
X_{N}^{T}
\end{array}\right)=\left(\begin{array}{ccc}
x_{11} & \cdots & x_{1 N} \\
\vdots & \ddots & \vdots \\
x_{N 1} & \cdots & x_{N N}
\end{array}\right), \quad Y=\left(y_{1}, \ldots, y_{N}\right)^{T}
$$

and the training data $\left\{\left(\boldsymbol{X}_{1}, y_{1}\right), \ldots,\left(\boldsymbol{X}_{N}, y_{N}\right)\right\}$, the LASSO coefficients are estimated based on the following argument:

$$
\hat{\beta}_{\text {lasso }}=\arg \min _{\beta}\left\{\sum_{i=1}^{N}\left(y_{i}-\beta_{0}-\sum_{j=1}^{d} \beta_{i} x_{i j}\right)^{2}\right\} \quad \text { subject to } \sum_{j=1}^{d}\left|\beta_{j}\right| \leq k, k>0
$$

The parameter $k$ is called 'tuning parameter' and controls the amount of shrinkage applied to the coefficients (Tibshirani, 2011). For more details on the mathematical specifications of LASSO see Wang et al. 2007.

In this study, a sensitivity analysis is carried out for selecting the optimal value of $k$ based on the in-sample period. Therefore our final constraint is:

$$
\left|\beta_{M L P}\right|+\left|\beta_{R N N}\right|+\left|\beta_{P S N}\right|+\left|\beta_{R B F N N}\right| \leq 17.2
$$

Subject to the above coefficient constraint, the final LASSO forecast combinations are given by the equation:

$$
f_{c_{N N S}}^{t}=0.86 f_{M L P}^{t}+2.68 f_{R N N}^{t}+4.44 f_{P S N}^{t}+7.67 f_{R B F N N}^{t}+\varepsilon_{t}
$$

The use of the constraint creates a penalization balance on each estimate and leads some coefficients to zero or close to zero. In that way, the result is more adaptive than a simple regression.

\subsection{Kalman Filter}


Kalman Filter is an efficient recursive filter that estimates the state of a dynamic system from a series of incomplete and noisy measurements (Wells 1996). In this application, we suggest the use of Kalman Filter as a time-varying coefficient combination forecast. In order to define the recursive algorithm, we need a measurement equation to combine the forecasts and a state equation to update the weights of the combination at each step. Those equations are given below.

Measurement Equation: $f_{c_{N N S}}^{t}=\sum_{i=1}^{4} a_{i}^{t} f_{i}^{t}+\varepsilon_{t}, \quad \varepsilon_{t} \sim N I D\left(0, \sigma_{\varepsilon}^{2}\right)$

State Equation: $\quad a_{i}^{t}=a_{i}^{t-1}+n_{t}, \quad n_{t} \sim N I D\left(0, \sigma_{n}^{2}\right)$

Where:

- $\quad f_{c_{N N S}}^{t}$ is the dependent variable (combination forecast) at time $t$

- $\quad f_{i}^{t}(i=1,2,3,4)$ are the independent variables (individual forecasts) at time $t$

- $\quad a_{i}^{t}(i=1,2,3,4)$ are the time-varying coefficients at time $t$ for each $\mathrm{NN}$

- $\quad \varepsilon_{t}, n_{t}$ are the uncorrelated error terms (noise)

The alphas are calculated by a simple random walk and we initialized $\varepsilon_{1}=0$. Following Hatemi-J and Roca (2006), our Kalman Filter model has as a final state the following:

$$
f_{c_{N N S}}^{t}=10.32 f_{M L P}^{t}+12.18 f_{R N N}^{t}+31.34 f_{P S N}^{t}+52.21 f_{R B F N N}^{t}+\varepsilon_{t}
$$

From the above equation, it is obvious that the Kalman filtering process favors the RBFNN model, which is the model that performs best individually.

\subsection{Support Vector Regression (SVR)}

Vapnik (1995) established Support Vector Regression (SVR) as a robust technique for constructing data-driven and non-linear empirical regression models. SVRs are commonly used in financial and macroeconomic applications (Ince and Trafalis 2008; Reboredo et al. 2012; Xu et al. 2013). Their advantages, such as providing global and 
unique solutions, not suffering from local minima and balancing model accuracy and model complexity are well documented in literature (Suykens et al. 2002; Lu et al. 2009). A simple SVR function can be specified as:

$$
f(x)=w^{T} \varphi(x)+b
$$

where $w$ and $b$ are the regression parameter vectors of the function and $\varphi(x)$ is the nonlinear function that maps the input data vector $x$ into a feature space where the training data exhibit linearity (see figure 3c).

\section{[Insert Figure 3]}

The $\varepsilon$-sensitive loss $L_{\varepsilon}$ function finds the predicted points that lie within the tube created by two slack variables $\xi_{i}, \xi_{i}^{*}$ (see figure $3 a$ and $\left.3 b\right)$ :

$$
L_{\varepsilon}\left(x_{i}\right)=\left\{\begin{array}{c}
0 \text { if }\left|y_{i}-f\left(x_{i}\right)\right| \leq \varepsilon \\
\left|y_{i}-f\left(x_{i}\right)\right|-\varepsilon \text { if other }
\end{array}, \varepsilon \geq 0\right.
$$

$L_{\varepsilon}$ finds the predicted values that have at most $\varepsilon$ deviations from the actual obtained values $y_{i}$. Therefore, $\varepsilon$ quantifies the degree of model noise insensitivity. The goal is to solve the following argument:

Minimize $C \sum_{i=1}^{n}\left(\xi_{i}+\xi_{i}^{*}\right)+\frac{1}{2}\|w\|^{2}$ subject to $\left\{\begin{array}{l}\xi_{i} \geq 0 \\ \xi_{i}^{*} \geq 0 \\ C>0\end{array}\right\}$ and $\left\{\begin{array}{l}y_{i}-w^{T} \varphi\left(x_{i}\right)-b \leq+\varepsilon+\xi_{i} \\ w^{T} \varphi\left(x_{i}\right)+b-y_{i} \leq+\varepsilon+\xi_{i}^{*}\end{array}\right\}$

Equation (16) attempts to minimize the sum of the norm term $\|w\|^{2}$ and the $\operatorname{term}\left\{\sum_{i=1}^{n}\left(\xi_{i}+\xi_{i}^{*}\right)\right\}$. The first term characterizes the complexity of the model, while the second is the training error, as specified by the slack variables. The parameter $C$ satisfies the need to trade model complexity for training error and vice versa (Cherkassky and Ma 2004). The above solution is based on the introduction of two Lagrange multipliers $a_{i}, a_{i}^{*}$ and mapping with a kernel function $K\left(x_{i}, x\right)$ :

$$
f(x)=\sum_{i=1}^{n}\left(a_{i}-a_{i}^{*}\right) K\left(x_{i}, x\right)+b \text { where } 0 \leq a_{i}, a_{i}^{*} \leq C
$$


The application of the kernel function transforms the original input space into one with more dimensions, where a linear decision border can be identified. The original input space consists of four vectors. These vectors correspond to the individual forecasts of MLP, RNN, PSN and RBFNN derived from the empirical simulation of section 3.3. The extended mathematical explanation of this solution can be found in Vapnik (1995).

Choosing the $\varepsilon$ parameter is indeed a challenging task, because it depends on the noise of the training datasets. In practice, there are no optimal solutions to this problem. The majority of the researchers adopt the cross-validation approach (Cao et al. 2003; Duan et al. 2003). Hence, we apply the same procedure to our study. Another challenge is the selection of the kernel function. RBF kernels are popular in similar SVR applications, because they efficiently overcome overfitting and seem to excel in directional accuracy (Kim and Sohn 2010; Yu and Yao 2013). The four NN forecasts are used as inputs for a RBF $\varepsilon$-SVR simulation. The RBF kernel is specified as:

$$
K\left(x_{i}, x\right)=\exp \left(-\gamma\left\|x_{i}-x\right\|^{2}\right), \gamma>0
$$

From equations (16) and (18) it is obvious that we need to determine two kernelindependent parameters ( $\varepsilon$ and $C$ ) and the RBF parameter $(\gamma)$. This is achieved by a 5 -fold cross validation in our in-sample dataset, following Duan et al. 2003. The final single SVR forecast combination is calculated with the following optimized set of parameters $\varepsilon=0.15, \gamma=4.18$ and $C=94.8$. The out-of-sample observations of UNEMP time series are not used at all for tuning our SVR model.

\section{Empirical Results}

As it is standard in literature, in order to evaluate statistically our forecasts, the RMSE, the MAE, the MAPE and the Theil-U statistics are computed. For all four of the error statistics retained the lower the output, the better the forecasting accuracy of the model concerned. The mathematical formulas of these statistics are given in Appendix B. In Table 3 we present the statistical performance of all our models in the in-sample period.

[Insert Table 3] 
From the above table it is obvious that from our individual forecasts, the RBFNN statistically outperforms all other models. All forecast combination techniques improve the forecasting accuracy. SVR is the superior model regarding all four statistical criteria. It would be interesting to see if the in-sample performance coincides with the out-ofsample one. Table 4 below summarizes the statistical performance of our models in the out-of-sample period.

[Insert Table 4]

The results of table 4 suggest that the statistical performance of the models in the out-ofsample period is consistent with the in-sample one and their ranking remains the same. All NN models outperform the traditional ARMA and STAR models. In addition, the RBFNN outperforms significantly the MLP and RNN in terms of statistical accuracy. The second best individual performance is presented by PSN, which remains less accurate than RBFNN. This means that the RBFNN manages to overcome the statistical performance of the traditional MLP and RNN, but also of PSN which in general has fast learning and powerful mapping abilities. The forecast combination techniques are all improving the accuracy of the individual performances. Even the least sophisticated Simple Average presents lower values in all four statistics in comparison with the best individual model, the RBFNN. The LASSO achieves higher forecast accuracy than Simple Average, but it does not perform better from the Kalman Filter and SVR. In this forecasting competition, SVR remains the superior model 'beating' Kalman Filter in every statistic in the out-of-sample period.

The statistical superiority of our best proposed architecture, namely the SVR, is confirmed by the Modified Diebold-Mariano (MDM) statistic as proposed by Harvey et al. (1997). The null hypothesis of the test is the equivalence in forecasting accuracy between couples of forecasting models. The MDM test ${ }^{3}$ is an extension of the DieboldMariano (1995) test and its statistic (DM) is presented below:

$$
M D M=T^{-1 / 2}\left[T+1-2 k+T^{-1} k(k-1)\right]^{1 / 2} D M
$$

\footnotetext{
${ }^{3}$ The MDM test follows the student distribution with T-1 degrees of freedom.
} 
where $T$ the number of the out-of-sample observations and $k$ the number of the step-ahead forecasts. In our case we apply the MDM test to couples of forecasts (SVR vs. another forecasting model). A negative realization of the MDM test statistic indicates that the first forecast (SVR) is more accurate than the second forecast. The lower the negative value, the more accurate are the SVR forecasts. The use of MDM test is common practice, because it assesses the significance of observed differences between the performances of two forecasts (Barhoumi et al., 2010). The statistic is measured in the out-of-sample period for the MSE and MAE loss functions. Table 5 below presents the values of the DM and MDM statistics for all the cases, comparing the SVR with its benchmarks.

\section{[Insert Table 5]}

The table shows that the MDM null hypothesis of equal forecasting accuracy is rejected for all comparisons and for both loss functions at the $1 \%$ confidence interval. The statistical superiority of the SVR forecasts is confirmed as the realizations of the MDM statistic are negative for both loss functions.

The results of this section support the idea of combining NN unemployment forecasts, since the Simple Average, LASSO, Kalman Filter and SVR present improve the statistical accuracy both in the in-sample and out-of-sample period. The fact that the insample statistical ranking of our NNs is consistent with the out-of-sample one proves that the training of our models is done effectively. The coefficient adaptivity of LASSO does not provide with such forecasting power to outperform the time-varying Kalman Filter process. Nonetheless, it is superior from all NNs and the less sophisticated Simple Average. SVR also confirms its forecasting superiority over all individual architectures and combining techniques. Finally, the fact that SVR is found always more accurate than Kalman Filters suggests that the adaptive trade-off between model complexity and training error of this technique seems more effective than the recursive ability of Kalman Filter to estimate the state of our process.

In general, the growing literature of NNs and more specifically of the utility of RBFNNs in similar forecasting exercises is extended. The improved statistical results of the Kalman Filter and SVR are supporting the evidence of previous studies that report the benefits of combining forecasts. In summary, the success of the non-linear and time- 
varying combination methods of this study indicates a need to experiment with more complex combination techniques and beyond traditional linear equivalents.

\section{Concluding Remarks}

This motivation of this study is to investigate the efficiency of the RBFNN in forecasting the US unemployment and explores the utility of Kalman Filter and SVR as forecast combination techniques. In terms of our RBFNN, an ARMA, a STAR and three different NNs, namely a MLP, RNN and PSN are used as benchmarks. Our forecast combination methods are benchmarked with a Simple Average and a LASSO. The statistical performance of our models is estimated throughout the period of 1972-2012, using the last seven years for out-of-sample testing.

The results show that the RBFNN statistically outperforms all models' individual performances. Even PSN which embodies fast learning abilities and powerful mapping capabilities cannot reach the RBFNN's levels of accuracy. The forecast combinations are successful, since both Kalman Filter and SVR techniques improve the statistical accuracy in comparison to the Simple Average and LASSO benchmarks. The Simple Average presents better results than all individual models, but it cannot outperform any of the more sophisticated combination methods. Finally, SVR is found to be the superior model of the forecasting competition, which is further confirmed by the modified Diebold Mariano test.

The idea of combining NN unemployment forecasts is promising, since the Simple Average, LASSO, Kalman Filter and SVR present improved statistical accuracy both in the in-sample and out-of-sample period. SVR is found always more accurate than Kalman Filter. This indicates that the adaptive trade-off between model complexity and training error of SVR is more effective from the recursive ability of Kalman Filter to estimate the state of our process. The general statistical performance of SVR allows us to conclude that it can be considered as an optimal forecast combination for the models and time series under study. The results are in line with the relevant literature which suggests that adaptive, time-varying, nonlinear models can be used to model macroeconomic series. Finally, The SVR and Kalman Filter forecast combinations could be further 
extended. A potential extension could be the use of individual forecasts from a larger pool of relevant models or the use of several macroeconomic indicators and different forecast horizons.

\section{Appendix}

\section{A. NNs' structure and training characteristics}

This appendix section briefly describes the structure of the three traditional NNs used to benchmark the RBFNN. It also includes a summary of the training characteristics of all four NNs.

Firstly, a typical MLP model is shown in the following figure.

$$
\text { [Insert figure A.1] }
$$

Where:

- $\quad x_{t}^{[n]}(n=1,2, \cdots, k+1)$ are the inputs (including the input bias node) at time $t$

- $\quad h_{t}^{[m]}(m=1,2, \ldots, j+1)$ are the hidden nodes outputs

- $\hat{Y}_{t}$ is the MLP output (target value)

- $\quad u_{j k}, w_{j}$ are the network weights

- $\int$ is the transfer sigmoid function $S(x)=\frac{1}{1+e^{-x}}$

- $\bigcirc$ is a linear function $F(x)=\sum_{i} x_{i}$

The Error Function to be minimized is:

$$
E\left(u_{j k}, w_{j}\right)=\frac{1}{T} \sum_{t=1}^{T}\left(Y_{t}-\hat{Y}_{t}\left(u_{j k}, w_{j}\right)\right)^{2}
$$

Secondly, the simple architecture of an RNN is presented below.

[Insert figure A.2] 
Where:

- $\quad x_{t}^{[n]}(n=1,2, \ldots, k+1), u_{t}^{[1]}, u_{t}^{[2]}$ are the RNN inputs at time $t$ (including bias node)

- $\tilde{y}_{t}$ is the output of the RNN

- $\quad d_{t}^{[f]}(f=1,2)$ and $w_{t}^{[n]}(n=1,2, \ldots, k+1)$ are the weights of the network

- $\quad U_{t}^{[f]}, f=(1,2)$ is the output of the hidden nodes at time $t$

- $\left(S\right.$ is the transfer sigmoid function : $S(x)=\frac{1}{1+e^{-x}}$

- $\bigcirc$ is a linear function: $F(x)=\sum_{i} x_{i}$

The Error Function to be minimized is:

$$
E\left(d_{t}, w_{t}\right)=\frac{1}{T} \sum_{t=1}^{T}\left(y_{t}-\tilde{y}_{t}\left(d_{t}, w_{t}\right)\right)^{2}
$$

Thirdly, figure A.3 describes the PSN architecture.

$$
\text { [Insert figure A.3] }
$$

Where:

- $\quad x_{t}(n=1,2, \ldots, k+1)$ are the model inputs

- $\quad y_{t}, \tilde{y}_{t}$ are the PSN input and output respectively

- $\quad w_{j}(j=1,2 . ., k)$ are the adjustable weights ( $k$ is the desired order of the network)

- $\quad$ The hidden layer activation function: $h(x)=\sum_{i} x_{i}$

- $\quad$ The output sigmoid activation function ( $c$ the adjustable term):

$$
\sigma(x)=\frac{1}{1+e^{-x c}}
$$

The Error Function minimized in this case:

$$
E\left(c, w_{j}\right)=\frac{1}{T} \sum_{t=1}^{T}\left(y_{t}-\tilde{y}_{t}\left(w_{k}, c\right)\right)^{2}
$$


Finally, the following table summarizes the training characteristics of the four NN architectures used in this forecasting competition.

[Insert Table A.1]

\section{B. Statistical Performance Measures}

The statistical performance measures are calculated as shown in table B.1 below.

[Insert Table B.1]

\section{References}

Barhoumi, K., Darné, O., Ferrara, L. et al. (2010). Are disaggregate data useful for factor analysis in forecasting French GDP? Journal of Forecasting, 29 (1-2), 132-144.

Bates, J.M., Granger, C.W.J. (1969) The Combination of Forecasts. Operational Research Society, 20 (4), 451-468.

Broomhead, D.S., Lowe, D. (1988). Multivariable functional interpolation and adaptive networks. Complex Systems, 2, 321-355.

Cao, L.J., Chua, K.S., Guan, L.K. et al. (2003). C-ascending support vector machines for financial time series forecasting. In: Computational Intelligence for Financial Engineering Proceedings, 317-323.

Chan,Y.L., Stock, J.H., Watson, M.W. (1999). A dynamic factor model framework for forecast combination. Spanish Economic Review, 1 (2), 91-121.

Chan, K.S., Tong, H. (1986). On estimating thresholds in autoregressive models. Journal of Time Series Analysis, 7 (3), 178-190.

Cherkassky, V., Ma, Y. (2004). Practical selection of SVM parameters and noise estimation for SVM regression. Neural Networks, 17 (1), 113-126. 
Deutsch, M., Granger, C.W.J., Teräsvirta, T. et al. (1994). The combination of forecasts using changing weights. International Journal of Forecasting, 10 (1), 47-57.

Diebold, F.X., Mariano, R.S. (1995). Comparing Predictive Accuracy. Journal of Business and Economic Statistics, 13 (1), 253-263.

Duan, K, Keerthi, S.S., Poo, A.N. et al. (2003). Evaluation of simple performance measures for tuning SVM hyperparameters. Neurocomputing, 51, 41-59.

Fernandes, M., Medeiros, M.C., Scharth, M. (2014). Modeling and predicting the CBOE market volatility index. Journal of Banking \& Finance, 40, 1-10.

Harvey, D., Leybourne, S., Newbold, P. et al. (1997). Testing the equality of prediction mean squared errors. International Journal of Forecasting, 13 (2), 281-291.

Hastie, T., Tibshirani, R., Friedman, J.H. (2009). The elements of statistical learning : data mining, inference, and prediction. $2^{\text {nd }}$ Ed. New York: Springer.

Hatemi-J., A., Roca, E. (2006). Calculating the optimal hedge ratio: constant, time varying and the Kalman Filter approach. Applied Economics Letters, 13 (5), 293-299.

Hiemstra, Y. (1996). Linear regression versus back propagation networks to predict quarterly stock market excess returns. Computational Economics, 9 (1), 67-76.

Huang, S.C., Wang, N.Y, Li, T.Y., Lee,Y.C., Chang, L.F., Pan, T.H. (2013). Financial Forecasting by Modified Kalman Filters and Kernel Machines. Journal of Statistics and Management Systems, 16, 2-03, 163-176.

Ince, H., Trafalis, T.B. (2008). Short term forecasting with support vector machines and application to stock price prediction. International Journal of General Systems, 37 (6), 677-687.

Kim, H.S., Sohn, S.Y. (2010) Support vector machines for default prediction of SMEs based on technology credit. European Journal of Operational Research, 201 (3), 838-846.

Kapetanios, G., Labhard, V., Price, S. (2008) Forecast combination and the Bank of England's suite of statistical forecasting models. Economic Modelling, 25 (4), 772-792. 
Koop, G., Potter, S.M. (1999) Dynamic Asymmetries in U.S. Unemployment. Journal of Business \& Economic Statistics, 17 (3), 298-312.

Lin, C.J., Teräsvirta T. (1994). Testing the Constancy of Regression Parameters against Continuous Structural Changes. Journal of Econometrics, 62 (2), 211-228.

Liang, F. (2005). Bayesian neural networks for nonlinear time series forecasting. Statistics and Computing,15 (1), 13-29.

Lu, C.J., Lee, T.S., Chiu, C.C. et al. (2009). Financial time series forecasting using independent component analysis and support vector regression. Decision Support Systems, 47 (2), 115-125.

Milas, C., Rothman, P. (2008). Out-of-sample forecasting of unemployment rates with pooled STVECM forecasts. International Journal of Forecasting, 24 (1) , 101-121.

Montgomery, A.L, Zarnowitz, V., Tsay, R.S., Tiao, G.C. et al. (1998). Forecasting the U.S. Unemployment Rate. Journal of the American Statistical Association, 93 (442), 478493.

Moshiri, S., Brown, L. (2004). Unemployment variation over the business cycles: a comparison of forecasting models. Journal of Forecasting, 23 (7), 497-511.

Moshiri, S., Cameron, N.E., Scuse, D. et al. (1999). Static, Dynamic, and Hybrid Neural Networks in Forecasting Inflation. Computational Economics, 14 (3), 219-235.

Newbold, P., Granger, C.W.J. (1974) Experience with Forecasting Univariate Time Series and the Combination of Forecasts. Journal of the Royal Statistical Society, 137 (2), 131-165.

Olmedo, E. (2014). Forecasting Spanish Unemployment Using Near Neighbor and Neural Net Techniques. Computational Economics, 43(2), 183-197.

Özkan, F. (2013). Comparing the forecasting performance of neural network and purchasing power parity: The case of Turkey. Economic Modelling, 31, 752-758.

Reboredo, J.C., Matías, J.M., Garcia-Rubio, R. et al. (2012). Nonlinearity in Forecasting of High-Frequency Stock Returns. Computational Economics, 40 (3), 245-264. 
Rothman P. (1998). Forecasting Asymmetric Unemployment Rates. The Review of Economics and Statistics, 80 (1), 164-168.

Sermpinis, G., Dunis, C., Laws, J., Stasinakis, C. et al. (2012)Forecasting and trading the EUR/USD exchange rate with stochastic neural network combination and time-varying leverage. Decision Support Systems, 54 (1), 316-329.

Shapiro, A.F. (2000). A Hitchhiker's guide to the techniques of adaptive nonlinear models. Insurance: Mathematics and Economics, 26 (2-3), 119-132.

Shin, Y., Ghosh, J., 1991. The Pi-Sigma Network: An efficient Higher-order Neural Networks for Pattern Classification and Function Approximation. Proceedings of International Joint Conference of Neural Networks, 1, 13-18.

Skalin, J., Teräsvirta, T. (2002). Modeling Asymmetries and Moving Equilibria in Unemployment Rates. Macroeconomic Dynamics, 6 (2), 202-241.

Sundberg, R. (2006). Shrinkage Regression. In: El-Shaarawi, A.H., Piergosh, W.W. (eds.), Encyclopedia of Environmetrics, John Wiley \& Sons, Ltd., 4, 1994-1998.

Suykens, J.A.K., Brabanter, J. D., Lukas, L., Vandewalle, L. et al. (2002). Weighted least squares support vector machines: robustness and sparse approximation Neurocomputing, 48 (1-4), 85-105.

Swanson, N.R., Zeng, T. (2001). Choosing among competing econometric forecasts: Regression-based forecast combination using model selection. Journal of Forecasting, 20 (6), 425-440.

Szpiro, G.G. (1997). A Search for Hidden Relationships: Data Mining with Genetic Algorithms. Computational Economics, 10 (3), 267-277.

Tenti, P. (1996). Forecasting foreign exchange rates using recurrent neural networks. Applied Artificial Intelligence, 10 (6), 567-581.

Teräsvirta, T., Dijk, K.V., Medeiros, M.C. (2005). Linear models, smooth transition autoregressions, and neural networks for forecasting macroeconomic time series: A reexamination. International Journal of Forecasting, 21 (4), 755-774. 
Tibshirani, R. (2011). Regression shrinkage and selection via the lasso: a retrospective. Journal of the Royal Statistical Society: Series B (Statistical Methodology), 73 (3), 273282.

Vapnik, V.N. (1995).The nature of statistical learning theory, Springer.

Vasnev, A., Skirtun, M., Pauwels, L. et al. (2013). Forecasting Monetary Policy Decisions in Australia: A Forecast Combinations Approach. Journal of Forecasting, 32 (2), 151-166.

Wang, H., Li, G., Jiang, G. (2007). Robust Regression Shrinkage and Consistent Variable Selection Through the LAD-Lasso. Journal of Business and Economic Statistics, 25 (3), 347-355.

Wells, C. (1996). The Kalman filter in finance. Dordrecht, Kluwer Academic Publishers.

Xu, W., Li, Z., Cheng, C., Zheng, T. et al. (2013). Data mining for unemployment rate prediction using search engine query data. Service Oriented Computing and Applications, 7 (1), 33-42.

Yu, L., Yao, X. (2013). A total least squares proximal support vector classifier for credit risk evaluation. Soft Computing, 17 (4), 643-650.

Zhang, G., Patuwo, B.E., Hu, M.Y. et al. (1998). Forecasting with artificial neural networks: The state of the art. International Journal of Forecasting, 14 (1), 35-62.

Zhang, G.P., Qi, M. (2005). Neural network forecasting for seasonal and trend time series. European Journal of Operational Research, 160 (2), 501-514. 


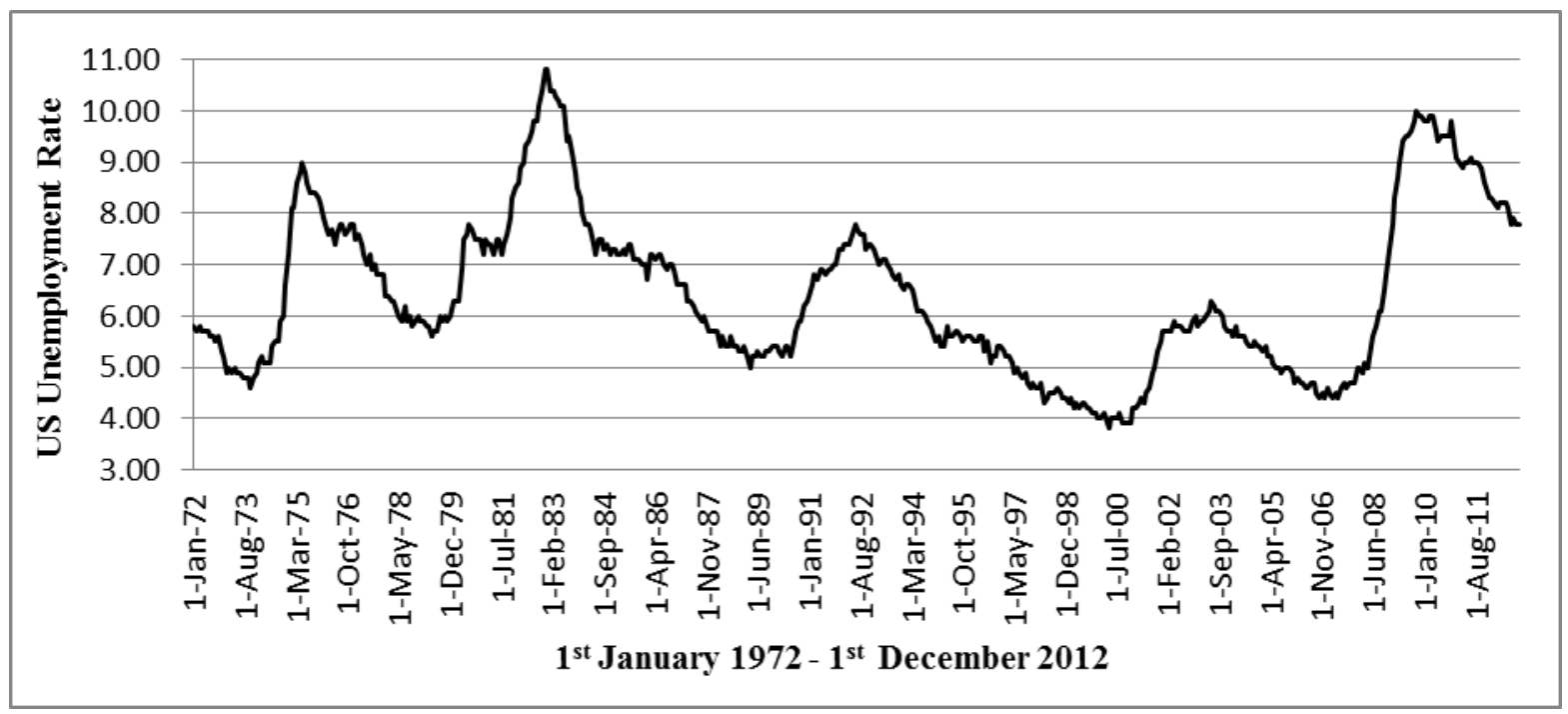

Figure 1: The US Unemployment Rate

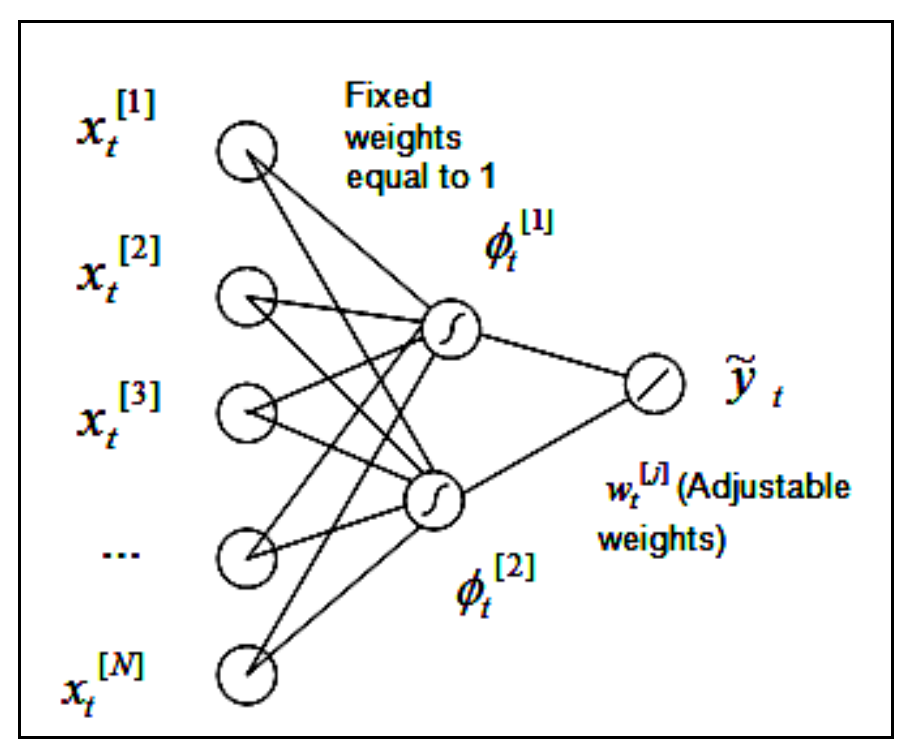

Figure 2: A RBF Neural Network with $\mathrm{N}$ inputs and 2 hidden nodes 


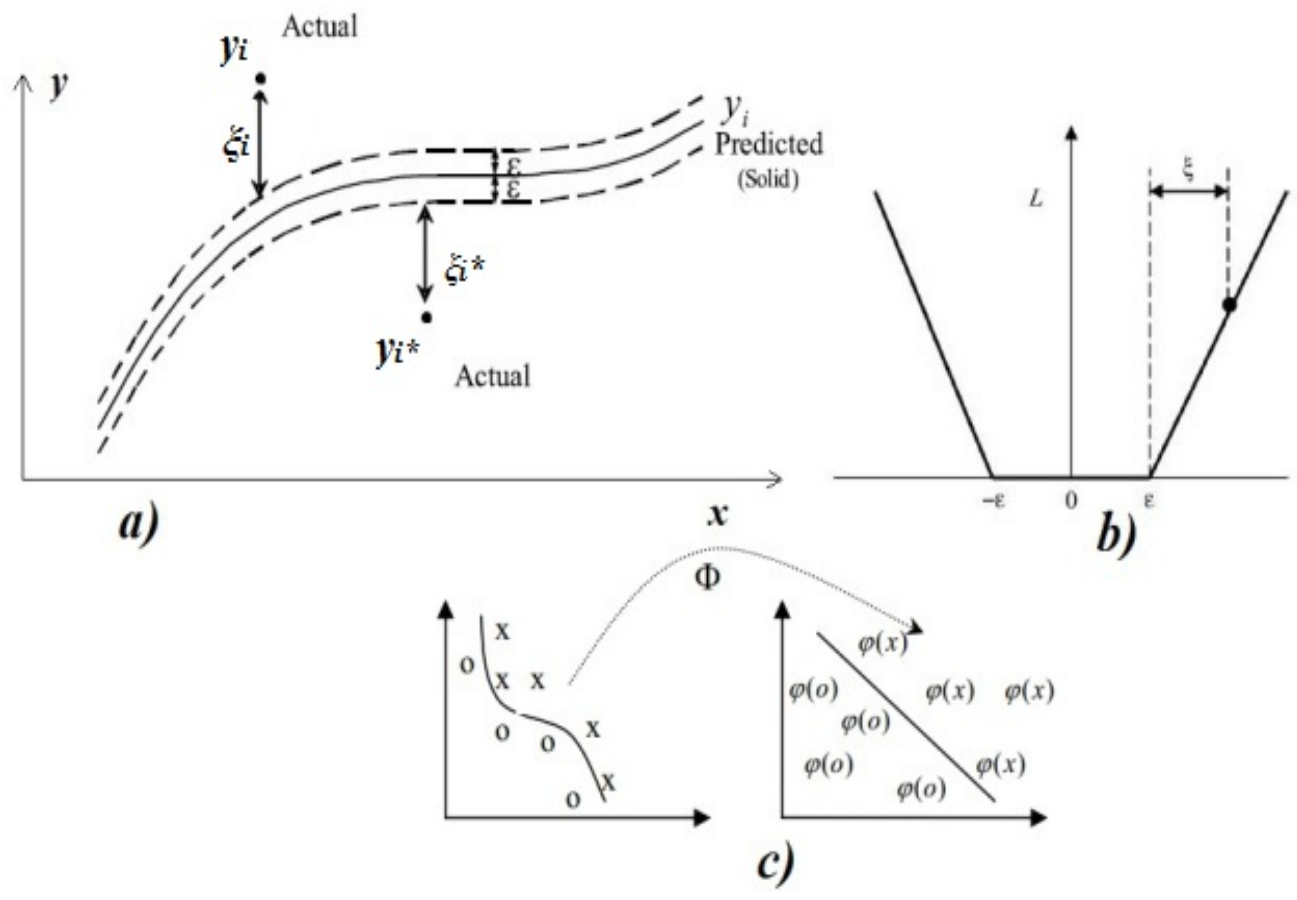

Figure 3: a) The $f(x)$ curve of SVR and the $\varepsilon$-tube, b) plot of the $\varepsilon$-sensitive loss function and c) mapping procedure by $\varphi(x)$

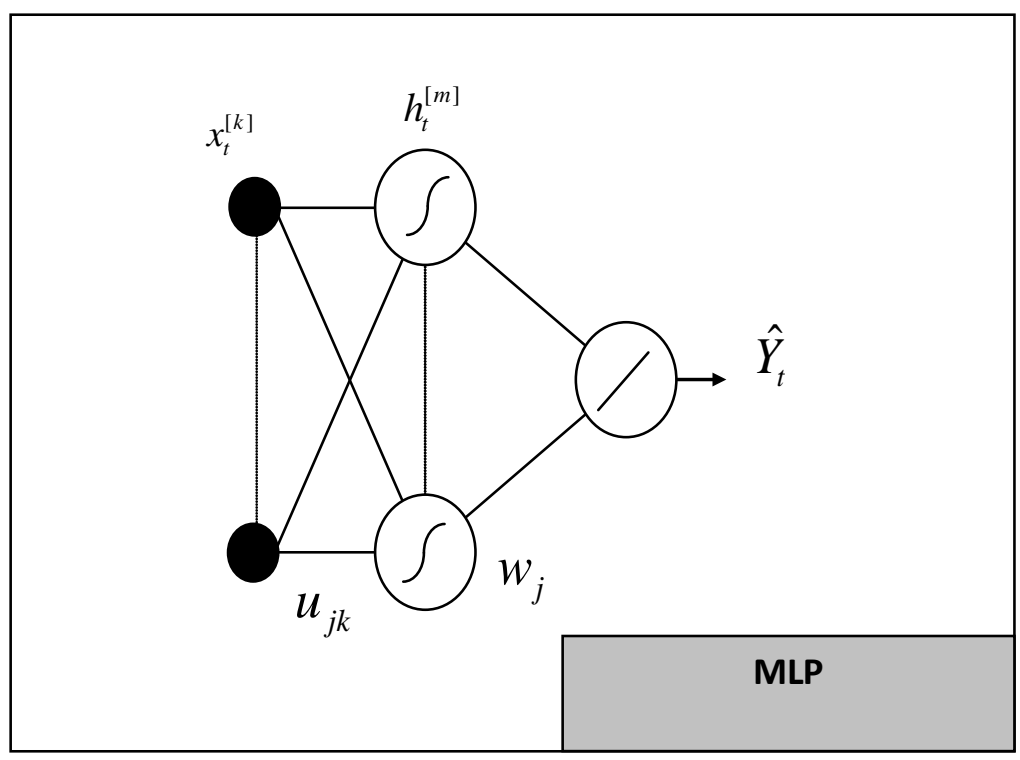

Figure A.1: A single output, fully connected MLP model (bias nodes are not shown for simplicity) 


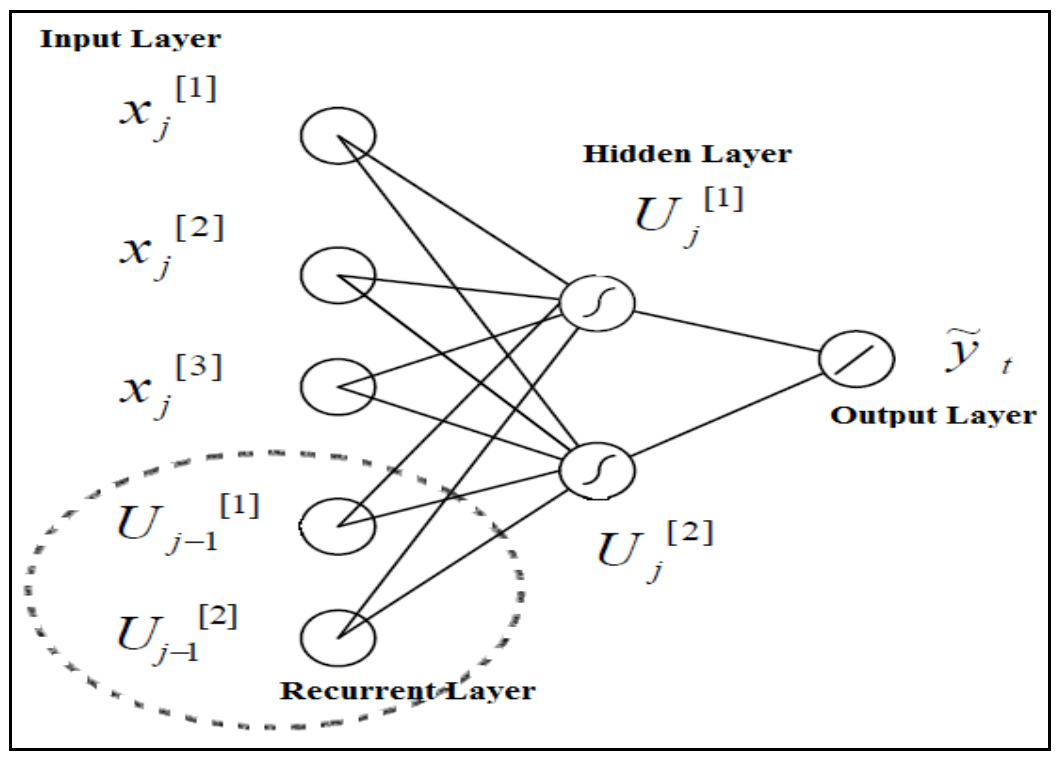

Figure A.2: RNN with two nodes in the hidden layer

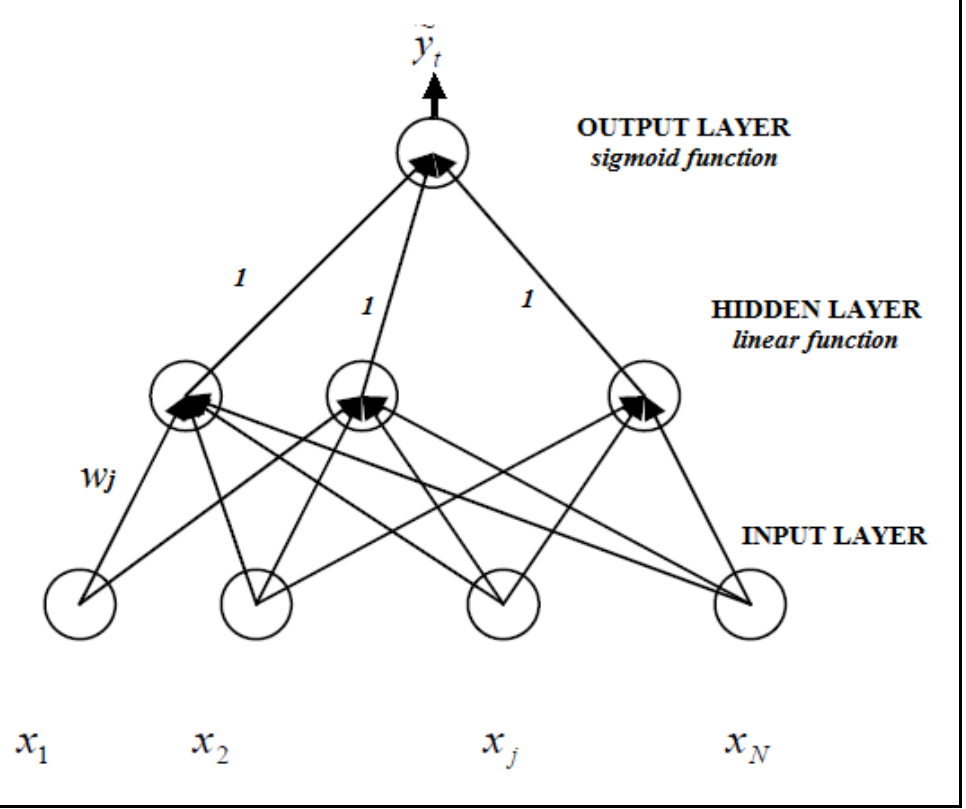

Figure A.3: A PSN with one output layer 


\begin{tabular}{|c|c|c|c|}
\hline PERIODS & MONTHS & START DATE & END DATE \\
\hline Total Dataset & 492 & $01 / / 01 / 1972$ & $01 / 12 / 2012$ \\
\hline $\begin{array}{c}\text { Training Dataset } \\
\text { (In-sample) }\end{array}$ & 324 & $01 / / 01 / 1972$ & $01 / 12 / 1998$ \\
\hline $\begin{array}{c}\text { Test Dataset } \\
\text { (In-sample) }\end{array}$ & 84 & $01 / 01 / 1999$ & $01 / 12 / 2005$ \\
\hline $\begin{array}{c}\text { Validation Dataset } \\
\text { (Out-of-sample) }\end{array}$ & 84 & $01 / 01 / 2006$ & $01 / 12 / 2012$ \\
\hline
\end{tabular}

Table 1: The US Unemployment Dataset - Neural Networks' Training Dataset

\begin{tabular}{|c|c|c|c|}
\hline MLP & RNN & PSN & RBFNN \\
\hline UNEMP (1)* & UNEMP (1) & UNEMP (1) & UNEMP (2) \\
\hline UNEMP (2) & UNEMP (3) & UNEMP (2) & UNEMP (3) \\
\hline UNEMP (4) & UNEMP (4) & UNEMP (3) & UNEMP (4) \\
\hline UNEMP (5) & UNEMP (6) & UNEMP (6) & UNEMP (7) \\
\hline UNEMP (7) & UNEMP (7) & UNEMP (8) & UNEMP (8) \\
\hline UNEMP (10) & UNEMP (9) & UNEMP (10) & UNEMP (9) \\
\hline UNEMP (11) & UNEMP (11) & - & UNEMP (11) \\
\hline UNEMP (12) & - & - & UNEMP (12) \\
\hline
\end{tabular}

* UNEMP (1) is the first autoregressive term of the UNEMP series

Table 2: Neural Networks’ Inputs

\begin{tabular}{|c|c|c|c|c|c|c|c|c|c|c|}
\cline { 2 - 11 } \multicolumn{1}{c|}{} & ARMA & STAR & MLP & RNN & PSN & RBFNN & Simple Average & LAS S O & Kalman Filter & S VR \\
\hline MAE & 1.9941 & 0.0094 & 0.0078 & 0.0077 & 0.0073 & 0.0068 & 0.0066 & 0.0065 & 0.0062 & 0.0058 \\
\hline MAPE & $65.25 \%$ & $60.27 \%$ & $52.78 \%$ & $50.17 \%$ & $47.73 \%$ & $44.38 \%$ & $43.02 \%$ & $41.58 \%$ & $40.78 \%$ & $38.52 \%$ \\
\hline RMSE & 2.5903 & 1.2105 & 1.0671 & 0.9572 & 0.9045 & 0.8714 & 0.8625 & 0.8556 & 0.8434 & 0.8216 \\
\hline Theil-U & 0.6717 & 0.6447 & 0.6142 & 0.5827 & 0.5325 & 0.5114 & 0.5021 & 0.4903 & 0.4717 & 0.4479 \\
\hline
\end{tabular}

Table 3: Summary of the In-Sample Statistical Performance

\begin{tabular}{|c|c|c|c|c|c|c|c|c|c|c|}
\cline { 2 - 11 } \multicolumn{1}{c|}{} & ARMA & STAR & MLP & RNN & PSN & RBFNN & Simple Average & LAS S O & Kalman Filter & S VR \\
\hline MAE & 0.0332 & 0.0099 & 0.0082 & 0.0081 & 0.0079 & 0.0075 & 0.0072 & 0.0071 & 0.0068 & 0.0061 \\
\hline MAPE & $67.45 \%$ & $64.27 \%$ & $53.17 \%$ & $51.97 \%$ & $49.38 \%$ & $47.41 \%$ & $47.02 \%$ & $45.14 \%$ & $44.32 \%$ & $40.12 \%$ \\
\hline RMSE & 2.4043 & 1.2412 & 1.1657 & 0.9954 & 0.9527 & 0.9114 & 0.8915 & 0.8706 & 0.8519 & 0.8327 \\
\hline Theil-U & 0.5922 & 0.6773 & 0.5954 & 0.5891 & 0.5618 & 0.5331 & 0.5241 & 0.5196 & 0.5023 & 0.4713 \\
\hline
\end{tabular}

Table 4: Summary of the Out-of-sample Statistical Performance 


\begin{tabular}{|c|c|c|c|c|c|c|c|c|c|}
\cline { 2 - 10 } \multicolumn{1}{c|}{} & ARMA & STAR & MLP & RNN & PS N & RBFNN & Simple Average & LAS SO & Kalman Filter \\
\hline $\mathbf{M D}_{\mathbf{1}}$ & -10.18 & -9.91 & -9.31 & -9.17 & -8.13 & -7.83 & -6.23 & -5.78 & -5.53 \\
\hline $\mathbf{M D}_{\mathbf{2}}$ & -14.06 & -12.42 & -10.58 & -9.96 & -9.81 & -8.63 & -7.11 & -6.92 & -6.83 \\
\hline $\mathbf{M D M}_{\mathbf{1}}$ & -10.24 & -9.97 & -9.37 & -9.23 & -8.18 & -7.88 & -6.27 & -5.81 & -5.56 \\
\hline $\mathbf{M D M}_{\mathbf{2}}$ & -14.14 & -12.49 & -10.64 & -10.02 & -9.87 & -8.68 & -7.15 & -6.96 & -6.87 \\
\hline
\end{tabular}

Note: $M D_{1}, M D M 1$, and $M D_{2}, M D M_{2}$ are the statistics computed for the MSE and MAE loss function respectively.

Table 5: Summary results of Modified Diebold-Mariano statistics for MSE and MAE loss functions

\begin{tabular}{|c|c|c|c|c|}
\hline Parameters & MLP & RNN & PSN & RBFNN \\
\hline $\begin{array}{c}\text { Learning } \\
\text { algorithm }\end{array}$ & $\begin{array}{c}\text { Gradient } \\
\text { descent }\end{array}$ & $\begin{array}{c}\text { Gradient } \\
\text { descent }\end{array}$ & $\begin{array}{c}\text { Gradient } \\
\text { descent }\end{array}$ & $\begin{array}{c}\text { Gradient } \\
\text { descent }\end{array}$ \\
\hline Learning rate & 0.005 & 0.003 & 0.002 & 0.003 \\
\hline Momentum & 0.007 & 0.005 & 0.006 & 0.005 \\
\hline Iteration steps & 60000 & 50000 & 75000 & 45000 \\
\hline $\begin{array}{c}\text { Initialisation } \\
\text { of weights }\end{array}$ & $\mathrm{N}(0,1)$ & $\mathrm{N}(0,1)$ & $\mathrm{N}(0,1)$ & $\mathrm{N}(0,1)$ \\
\hline Input nodes & 8 & 7 & 6 & 8 \\
\hline Hidden nodes & 6 & 6 & 5 & 4 \\
\hline Output node & 1 & 1 & 1 & 1 \\
\hline
\end{tabular}

Table A.1: The NNs training characteristics

\begin{tabular}{|c|c|}
\hline STATISTICAL PERFOMANCE MEASURES & DESCRIPTION \\
\hline Mean Absolute Error & $\begin{array}{c}\text { MAE }=\left(\frac{1}{n}\right) \sum_{\tau=t+1}^{t+n}\left|\hat{Y}_{\tau}-Y_{\tau}\right| \\
\hat{Y}_{\tau} \text { the forecasted value } Y_{\tau} \text { being the actual value and }\end{array}$ \\
\hline Mean Absolute Percentage Error & MAPE $=\frac{1}{n} \sum_{\tau=t+1}^{t+n}\left|\frac{Y_{\tau}-\hat{Y}_{\tau} \mid}{Y_{\tau}}\right|$ \\
\hline Root Mean Squared Error & RMSE $=\sqrt{\frac{1}{n} \sum_{\tau=t+1}^{t+n}\left(\hat{Y}_{\tau}-Y_{\tau}\right)^{2}}$ \\
\hline Theil-U & Theil $-U=\frac{\sqrt{\left(\frac{1}{n} \sum_{\tau=t+1}^{t+n}\left(\hat{Y}_{\tau}-Y_{\tau}\right)^{2}\right.}}{\sqrt{\frac{1}{n} \sum_{\tau=t+1}^{t+n} \hat{Y}_{\tau}^{2}}+\sqrt{\frac{1}{n} \sum_{\tau=t+1}^{t+n} Y_{\tau}^{2}}}$ \\
\hline
\end{tabular}

Table B.1: Statistical Performance Measures and Calculation 